\title{
Tactile motion lacks momentum
}

Gianluca Macauda ${ }^{1,4}$, Bigna Lenggenhager ${ }^{1,2}$, Rebekka Meier ${ }^{1}$, Gregory Essick ${ }^{3}$ Peter Brugger $^{1,2}$

${ }^{1}$ Neuropsychology Unit, Department of Neurology, University Hospital Zurich,

Zurich, Switzerland

${ }^{2}$ Center for Integrative Human Physiology (ZIHP), University of Zurich, Zurich, Switzerland

${ }^{3}$ School of Dentistry, University of North Carolina, Chapel Hill, NC USA

${ }^{4}$ Department of Psychology, University of Bern, Bern, Switzerland

Corresponding author:

Gianluca Macauda,

Neuropsychology Unit

Department of Neurology,

University Hospital Zurich,

CH-8091 Zurich;

phone ++41 4425555 79;

e-mail: gianluca.macauda@psy.unibe.ch

Key words: representational momentum, tactile motion, illusion, somatosensory system 


\section{Abstract}

The displacement of the final position of a moving object in the direction of the observed motion path, i.e. an overestimation, is known as Representational Momentum. It has been described both in the visual and auditory modality, and is suggested to be modality-independent. Here, we tested whether a Representational Momentum can also be demonstrated in the somatosensory domain. While the cognitive literature on Representational Momentum suggests that it can, previous work on the psychophysics of tactile motion perception would rather predict an underestimation of the perceived endpoint of a tactile stimulus. Tactile motion stimuli were applied on the left and the right dorsal forearms of 32 healthy participants, who were asked to indicate the subjectively perceived endpoint of the stimulation. Velocity and direction of the trajectory was varied. Contrary to the prediction based on the Representational Momentum literature, participants in our experiment significantly displaced the endpoint against the direction of movement (underestimation). The results are thus compatible with previous psychophysical findings on the perception of tactile motion. Further studies combining paradigms from classical psychophysics and cognitive psychology will be needed to resolve the apparently paradoxical predictions by the two literatures. 


\section{Introduction}

In the cognitive literature, the term representational momentum (RM) refers to the displacement of the final position of a moving object in the direction of the observed or anticipated motion path usually presented in the visual domain (Freyd \& Finke, 1984; Hubbard \& Bharucha, 1988). The term is chosen in analogy to the physical momentum (Hubbard, 2014), as some factors of the physical world such as velocity (e.g. Freyd \& Finke, 1984) andimplied gravity (Hubbard, 1990, 1995b, 1995c) have been shown to influence the size of RM. RM can be regarded as point in case of dynamic representations of the perceived external world, even when the presented stimuli solely imply motion. This is the case, for instance, when two static photographs of a moving object are flashed in temporal succession and may then suggest a specific direction of motion. It is argued that such dynamic mental representations are important to better survive in a dynamic environment (Freyd, 1992). This is also in line with current theories of predictive coding which advocate a top-down structure of perception based on generative internal (dynamic) models (e.g. Hohwy, 2013). Thus, RM is considered a higher-level phenomenon (for exhaustive reviews see Hubbard, 2005, 2014). This suggests that the effect should be modality independent, which seems confirmed by the observation of a RM also for auditory motion or pitch changes (Freyd, Kelly, \& DeKay, 1990; Getzmann \& Lewald, 2009; Getzmann, Lewald, \& Guski, 2004; Hubbard, 1995a; Johnston \& Jones, 2006). However, the notion of RM being a higher level phenomenon has been disputed as other studies focusing on other dimensions of dynamic changes, for instance in brightness (Brehaut \& Tipper, 1996) or facial expressions (Thornton, 2014; Yoshikawa \& Sato, 2008) have not always found an RM-like effect. In this context Kerzel (2002) has shown that the occurrence of RM is strongly dependent on the target's predictability.

The absence of relevant studies of RM in extra-visual domains appears particularly puzzling in the case of touch, as there is a vast literature on tactile motion processing (Essick, 1998; Essick et al., 2010; Whitsel et al., 1986), and tactile and visual motion have recently been out- 
lined as using highly similar canonical computations (Pack \& Bensmaia, 2015). Especially in the field investigating non-veridical perceptions, crossmodal comparisons may be helpful to uncover underlying mechanisms (Christopher Bill \& Teft, 1972; Helson, 1930; Sarrazin, Giraudo, \& Pittenger, 2005). Evidence of a tactile RM would allow to disentangle body-centered and space-centered frames of references, which is relevant for patients with spatial processing deficits, in whom RM has previously been shown to be altered (Lenggenhager et al., 2012). Against this background, we set out to test whether RM is reproducible in the somatosensory domain.

We investigated in healthy participants where on the skin they would perceive the endpoint of a trajectory of stimuli being moved along their right or left arms with different velocities and directions. We considered two alternative hypotheses stemming from two different literatures. A) If RM is a genuinely supramodal effect and the "tactile RM" thus postulated follows the basic properties of its analogues in the visual and auditory modalities, we would expect the experienced endpoint of a motion on the skin to be displaced in the direction of the movement, and increasingly so with increasing velocities. In line with this hypothesis, in a recently described tactile illusion, we found that a motion on the volar forearm lead to an overestimation of the perceived motion path length (Brugger \& Meier, 2015).

B) However, some observations from the literature on tactile motion perception would suggest a more complex pattern of findings, i.e. a mislocalization of tactile motion in direction of movement (overestimation) with slow stimuli and a decrease in perceived distance with increasing stimulus velocity (underestimation; Whitsel et al., 1986 for the dorsal forearm). Such a finding would predict an absent, or reverse RM at least for relatively fast tactile movements.

\section{Methods}

\section{Participants}

Thirty-two healthy volunteers participated in this study (16 female, mean age $=52.8, S D=$ 14.1). All participants were right-handed according to the inventory by Chapman and Chap- 
man (1987) and a performance test by Tapley and Bryden (1985). None of the participants reported a history of neurological or psychiatric disorders. The study was approved by the local Ethics Committee of the University of Basel. All participants gave written informed consent before the experiment.

\section{Procedure}

First, participants were instructed about the experimental procedure and filled in questionnaires regarding medical history and handedness, and performed the handedness test by Tapley and Bryden (1985). RM for the left and right arm was established with half of the participants starting with the left and the other with the right arm. Participants sat in front of a table and positioned their lower arms perpendicular to the upper arm on a cushion and held a stylus with the free, contralateral hands (see Fig. 1). Subsequently, participants were instructed to turn their heads away from the stimulated arm and close their eyes. The experimenter manually applied 24 motion paths on the participants' lower arms. Stimuli were delivered with the aid of a stylus $(26.5 \mathrm{~cm})$, and the motion differed in velocity, length and direction (proximodistal versus distal-proximal). They were applied always by the same experimenter (author R.Meier). To standardize the lines on the skin, two color-coded plates made of cardboard with the lengths $8 \mathrm{~cm}$ and $16 \mathrm{~cm}$ were used. These were not visible to the participants at any time of the experiment nor did the plates ever touch the participants' skin. They assisted the experimenter to apply different combinations of velocity, direction and length in a pseudorandom order (indicated on a protocol sheet), which was identical for each participant. Two different velocities of tactile stimulation $(6 \mathrm{~cm} / \mathrm{s}$ and $27 \mathrm{~cm} / \mathrm{s})$ were selected based on a range of velocities used in previous experiments on the lower arm (Essick, Bredehoeft, McLaughlin, \& Szaniszlo, 1991). These velocities are at the extremes of a velocity range over which distance was perceived to be invariant with movement velocity in Whitsel et al. (1986). After each stimulation, participants were asked to open their eyes and to indicate the felt endpoint of the felt motion path with the stylus (see Fig. 1 for the setup). The whole procedure was videotaped 
for offline analysis. The camera and tripod were put on a fixed position on the table, with the camera facing the participants' lower arms from above in a fix distance from an individual arm's surface. The deviation of a participant's felt endpoint from the actual endpoint of stimulus motion was measured offline on screen to the nearest mm.

\section{*** PLEASE INSERT FIGURE 1 HERE ***}

\section{Analysis}

Analyses were run using the statistical programming language R version 3.0.2 (R Core Team, 2013) including the yarrr (Phillips, 2016), tidyverse (Wickham, 2016) and ez package (Lawrence, 2016). Data points are always relative to the actual stopping point. Positive values represent a mislocalization in direction of motion, i.e. an RM (overestimation), while negative values correspond to a displacement opposite to the direction of motion (underestimation). Data and analysis script are publicly available on the Openscience Framework (osf.io/wx776). Outliers were defined as values above or below three standard deviations from the mean for each measured variable and excluded from the analysis. For the overall mean of the RM data a two-tailed one sample t-test was calculated and compared to a mean of zero (i.e., no displacement). An ANOVA with within-subject factors Arm (right, left) $\times$ Velocity (fast, slow) $\times$ Direction (proximal, distal) $\times$ Length (short, long) was calculated.

\section{Results}

Several outliers in the different variables were attributed to only one participant. Hence, this participant was excluded, resulting in a sample of $n=31$, 15 female, mean age $=52.1 \pm 13.7$. A simple t-test showed that the mean of each participant over all conditions was significantly different from 0 (mean $=-12.90, S D=7.33, t_{(30)}=-9.80, p<.001, d=-1.76$ Fig. 2 ), indicating that there was an underestimation. The repeated measures ANOVA with factors Arm $\times$ Velocity $\times$ Direction $\times$ Length resulted in significant main effects of Velocity $\left(F_{(1,30)}=46.53\right.$, $\left.p<.001, \eta^{2}=.065\right)$, with faster stimuli producing more underestimation, Direction $\left(F_{(1,30)}=\right.$ 
18.08, $\left.p<.001, \eta^{2}=.13\right)$, with more underestimation by the wrist, and Length $\left(F_{(1,30)}=10.17\right.$, $p=.003, \eta^{2}=.018$ ), with longer distances leading to more underestimation, and significant interaction effect of Length $\times$ Direction $\left(F_{(1,30)}=7.62, p=.01, \eta^{2}=.006\right)$. The interaction indicated a larger underestimation when the tactile motion was proximo-distal. Also, in the proximo-distal condition, no difference between long and short motion stimuli was apparent, but in the distal-proximal condition short motion stimuli showed the least underestimation. . Moreover, the interaction Velocity $\times$ Direction $\times$ Length $\left(F_{(1,30)}=13.38, p<.001, \eta^{2}=.006\right)$ was also significant (Fig 2) and reflected an attenuation of the Length $\times$ Direction interaction for the slow moving stimuli. Underestimation was greatest for distally directed fast motion.

\section{*** PLEASE INSERT FIGURE 2 HERE ***}

\section{Discussion}

We set out to investigate RM in the tactile modality and found participants to displace the perceived endpoint towards the starting point of the motion, i.e an underestimation. This finding was especially pronounced for longer and fast motion trajectories. The pattern of results is thus contrary to the simple prediction, based on RM in the visual modality, that the perceived endpoint would be displaced in the direction of motion. Instead, we have replicated some findings of the previous literature on tactile motion perception, whose relevance for a crossmodal comparison of perceived motion (in the visual fields vs. on the skin) has never been considered.

In two experiments similar to ours but not designed in the framework of RM, Whitsel and colleagues (1986) showed significant underestimation. These authors described a decrease in perceived distance with increasing stimulus velocity, an observation first reported in 1885 (Hall \& Donaldson, 1885) and later also by Langford, Hall \& Monty (1973). In Whitsel and colleagues' (1986) experiments, the effect of tactile velocity on subjective perception was assessed meticulously using a range of velocities from 1 to $256 \mathrm{~cm} / \mathrm{s}$. It was found that only 
the slowest tested velocity $(1 \mathrm{~cm} / \mathrm{s})$ led to a misperception of the endpoint in the direction of motion - analogous to a RM and reminiscent of a recently published illusion, in which a distal-proximal tactile motion starting at the wrist leads to an anticipation of the perceived touch of the elbow crook (Brugger \& Meier, 2015). With increasing velocity, however, the tactile path length perception changed into an underestimation. The decrease in experienced path length was not linear but interrupted by a plateau at velocities $5-25 \mathrm{~cm} / \mathrm{s}$, where there was no relationship between velocity and perceived endpoint. In our study we applied stimulus velocities at the borders of this range (6 and $27 \mathrm{~cm} / \mathrm{s}$ ) and, similar to Whitsel et al. (1986), found a main effect of velocity on subjective distance with faster stimuli perceived more in the direction of the starting point. However, there are notable methodological differences between the two studies.

While in the experiments by Whitsel and colleagues (1986), participants had to make an explicit estimate of the length of the stimulation path with the aid of a visual figure, our measure was selected to match the methodology of the classical RM, i.e. in visual paradigms. Another difference to the experiment by Whitsel et al. (1986) concerns stimulus application. While these authors used robot-controlled tactile stimulation, we relied on manually applied touch. It cannot be excluded that the situation of interpersonal touch leads to a neural response that is different from that of automated stimulation (Gallace \& Spence, 2010). In the illusion experiment by Brugger and Meier (2015), mentioned above, a tactile motion stimulus elicited an RM-like effect, but there the endpoint was predefined (crook of the elbow) and participants had to indicate when this endpoint was reached. The conditions in their experiment can thus not readily be compared with the conditions employed here.

The magnitude of the mislocalization error in the present study is in line with previous findings in slightly different paradigms. Using dynamic tactile stimuli Seizova-Cajic and Taylor (2014) found a systematic mislocalization bias towards the central regions of the forearm. This tendency would fit with the results of the present study. Interestingly, when no motion 
was present, the elbow and wrist served as 'anchors', resulting in an elongated representation of the forearm (Trojan et al., 2006), of potential interest also for the findings by Brugger and Meier (2015). The elongated representation would be consistent with overestimated trajectories in the visual (e.g. Actis-Grosso \& Stucchi, 2003; Hubbard \& Motes, 2002) modalities. Together, these authors' findings suggest that tactile motion might be directly related to the mislocalization error also found in the present study, but that future studies of RM on the skin should ideally use both dynamic and static tactile stimuli.

Still different methodological setups may be relevant to the discussion of whether or not RM can be observed in the somatosensory modality or the sensorimotor system. Brouwer and colleagues (2004) presented participants with dumbbells of increasing or decreasing size and had them "grasp" the final size. They found significant RM in grip aperture between thumb and index finger. Although their study used visual stimulation, RM was not of the type of the classical mislocalization of the endpoint in a pointing response, but manifested itself in the postural-haptic modality. An effect related to RM is the flash-lag effect, which consists in the perceptual impression that a flash around a (non-fixated) moving object would lag behind the object (Nijhawan, 2002). It is known that when the object's movement is yoked to the observer's head or body movement (and hence, any retinal motion signal is absent) there is still a flash-lag effect (Cai, Jacobson, Baloh, Schlag-Rey, \& Schlag, 2000; Schlag, Cai, Dorfman, Mohempour, \& Schlag-Rey, 2000). This suggests that vestibular signals and postural information can lead to an underestimation response with respect to a visual stimulus. Finally, Nijhawan \& Kirschfeld (2003) described a cross-modal flash-lag effect in the visuo-motor system, i.e. the lagging behind of a flash aligned with the observer's invisible hand they voluntarily turned by the wrist. Recently, the same phenomenon has also been reproduced in the haptic modality, dubbed the buzz-lag effect (Cellini, Scocchia, \& Drewing, 2016). To the extent that the flash-lag effect may rest on RM of the invisible target (Hubbard, 2014), this find- 
ing would indicate that internal dynamic representation are valid in the sensory and motor system alike.

The underestimation we found in our data could either be related to lower-level processes, e.g. the neural representation of the forearm's receptive fields in somatosensory areas (Whitsel, Favorov, Kelly, \& Tommerdahl, 1991), or to some higher-level cognitive processes in the perception of velocity. It has been shown that velocity is underestimated in touch (Goldreich, 2007; Goldreich \& Tong, 2013; Tong, Ngo, \& Goldreich, 2016). These authors argue that such an underestimation could be explained in a Bayesian framework by biased velocity priors favoring slow motion and being valid for the visual, auditory and tactile modality alike (Senna, Parise, \& Ernst, 2015). This theory could explain our pattern of an increasing mislocalization of the endpoint towards the starting point with increasing velocity. The main effect of stimulation direction we found in our data, with proximal-distal stimulation leading to more mislocalization than distal-proximal stimulation would seem paradoxical in view of a higher locognosic acuity bythe wrist (Cody, Garside, Lloyd, \& Poliakoff, 2008), which would generally result in more noise by the wrist instead of specific underestimation. However, given the larger cortical representation of the wrist than elbow, proximal-distal movement results in increasingly greater neural activity and engagement of inhibition as the stimulus progresses along its path, thus perhaps explaining the larger underestimation at the wrist site. The results could also indicate the presence of another bias, one that is directed towards the torso. According to this account, a body or a mass could have an attracting influence to moving objects, similar to the influence of gravity. However, direct evidence for such an additional, directional "torso bias" is lacking, and its existence must remain speculative.

Other perceptual illusions in the visual modality, which can be found in analogous form also in the auditory and tactile modalities, suggest a canonical mechanism common to vision, audition and touch. For example, the Tau and Kappa effects are two complementary illusions in which either stimulus timing presentation or spatial intervals between two sources lead to an 
illusion of distance or timing (Christopher Bill \& Teft, 1972; Helson, 1930; Sarrazin et al., 2005). In experiments on the Tau effect, subjects systematically underestimated the spatial distance between stimuli separated by shorter temporal intervals (faster imputed velocities). Another illusion in the visual and haptic modality possibly related to RM is boundary extension, where visual scenes are remembered beyond their physical boundary, suggesting topdown influences (Intraub, 2004; Intraub, Morelli, \& Gagnier, 2015).

The contrasting finding of an underestimation could rest upon differences in the functionality of the three modalities. While the environment perceivable by vision and audition is potentially unlimited, the surface of the skin clearly defines the extent of tactile perception. The limited extent of the skin could serve as boundary, potentially provoking a rebound or cessation of the motion. Similarly, for visual stimuli it has been shown that a forward displacement decreased or even reversed when a target stimulus was approaching a boundary with the backward displacement increasing as a function of distance from said boundary (Hubbard \& Motes, 2005), dubbed “framing effect” in Lenggenhager et al. (2012, p. 1325). Thus, it is possible that the wrist and the elbow are perceived as boundaries at which the movement direction would be reversed. This theory could be tested in future experiments with a manipulation of the distance of the endpoint from the wrist and elbow. Likewise, as proximal-distal and distalproximal stimulations alternated (pseudo randomly) across the sequence of trials, participants may have built up a global sense of some oscillatory motion. This might have produced an underestimation response, as the trajectory of an oscillating object is typically underestimated (Cavanagh \& Anstis, 2013). Blockwise presentations of one motion direction could help to test this "oscillation hypothesis" in future experimentation.

Further studies using paradigms borrowed from both basic psychophysics and cognitive psychology will be needed to reconcile the apparent differences in motion perception across sensory modalities. These studies will reveal the nature of RM in the somatosensory modality and allow judgment of whether, in fact, tactile motion lacks momentum. 
A shortcoming of the present study could be that the response to the endpoint stimulation was given by the participant while visually inspecting the previously stimulated target skinsegment. This way the visual input could have influenced the somatosensory representation of distance, considering the observation that the spatial resolution of tactile perception improves when there is visibility of the touched body part (Kennett, Taylor-Clarke, \& Haggard, 2001). Furthermore, literature on tactile line bisections has suggested more accurate response for bisecting one’s own seen body parts than for external objects (Bolognini, Casanova, Maravita, \& Vallar, 2012), which could thus have increased precision in our study.

Future studies should carefully investigate purely visual RM observed on a body part, purely tactile RM felt on the same part and finally the combination of the two inputs in order to learn about any potential mutual benefits. Finally, eye movements should be assessed in all these cases as the role of ocular smooth pursuit could be related to RM (Kerzel, 2000, 2003; Kerzel, Jordan, \& Müsseler, 2001). Similarly, with eye fixation RM has been found to be weaker for continuous motion (Kerzel, 2003) as used in the present study, which may partially explain the findings.

\section{Acknowledgment}

We thank Stefan Engelter, senior neurologist University Hospital Basel, for encouraging us to publish this experiment. We thank Peter Rohner for the illustration of the experimental setup and Daniel Goldreich for his comments on a previous version of the manuscript. Finally, the very constructive critique of three expert reviewers is acknowledged.

\section{Ethical Statement}

Conflict of Interest: All authors declare that they have no conflict of interest.

Ethical approval: The study was approved by the local Ethics Committee of the University of Basel. All participants gave written informed consent before the experiment. 


\section{References}

Actis-Grosso, R., \& Stucchi, N. (2003). Shifting the start: backward mislocation of the initial position of a motion. Journal of Experimental Psychology. Human Perception and Performance, 29(3), 675-691.

Bolognini, N., Casanova, D., Maravita, A., \& Vallar, G. (2012). Bisecting real and fake body parts: effects of prism adaptation after right brain damage. Frontiers in Human Neuroscience, 6, 154. https://doi.org/10.3389/fnhum.2012.00154

Brehaut, J. C., \& Tipper, S. P. (1996). Representational momentum and memory for luminance. Journal of Experimental Psychology. Human Perception and Performance, 22(2), 480-501.

Brouwer, A.-M., Franz, V. H., \& Thornton, I. M. (2004). Representational momentum in perception and grasping: translating versus transforming objects. Journal of Vision, 4(7), 575-584. https://doi.org/10:1167/4.7.5

Brugger, P., \& Meier, R. (2015). A new illusion at your elbow. Perception, 44(2), 219 - 221. https://doi.org/10.1068/p7910

Cai, R. H., Jacobson, K., Baloh, R., Schlag-Rey, M., \& Schlag, J. (2000). Vestibular signals can distort the perceived spatial relationship of retinal stimuli. Experimental Brain Research, 135(2), 275-278. https://doi.org/10.1007/s002210000549

Cavanagh, P., \& Anstis, S. (2013). The flash grab effect. Vision Research, 91, 8-20. https://doi.org/10.1016/j.visres.2013.07.007

Cellini, C., Scocchia, L., \& Drewing, K. (2016). The buzz-lag effect. Experimental Brain Research, 234(10), 2849-2857. https://doi.org/10.1007/s00221-016-4687-4 
Chapman, L. J., \& Chapman, J. P. (1987). The measurement of handedness. Brain and Cognition, 6(2), 175-183.

Christopher Bill, J., \& Teft, L. W. (1972). Space-time relations: The effects of variations in stimulus and interstimulus interval duration on perceived visual extent. Acta Psychologica, 36(5), 358-369. https://doi.org/10.1016/0001-6918(72)90032-7

Cody, F. W. J., Garside, R. A. D., Lloyd, D., \& Poliakoff, E. (2008). Tactile spatial acuity varies with site and axis in the human upper limb. Neuroscience Letters, 433(2), 103108. https://doi.org/10.1016/j.neulet.2007.12.054

Essick, G. K. (1998). Factors affecting direction discrimination of moving tactile stimuli. ADVANCES IN PSYCHOLOGY-AMSTERDAM-, 127, 1-54.

Essick, G. K., Bredehoeft, K. R., McLaughlin, D. F., \& Szaniszlo, J. A. (1991). Directional sensitivity along the upper limb in humans. Somatosensory \& Motor Research, 8(1), $13-22$.

Essick, G. K., McGlone, F., Dancer, C., Fabricant, D., Ragin, Y., Phillips, N., ... Guest, S. (2010). Quantitative assessment of pleasant touch. Neuroscience and Biobehavioral Reviews, 34(2), 192-203. https://doi.org/10.1016/j.neubiorev.2009.02.003

Freyd, J. J. (1992). Dynamic representations guiding adaptive behavior. In Time, action and cognition (pp. 309-323). Springer. Retrieved from http://link.springer.com/chapter/10.1007/978-94-017-3536-0_32

Freyd, J. J., \& Finke, R. A. (1984). Facilitation of length discrimination using real and imaged context frames. The American Journal of Psychology, 97(3), 323-341.

Freyd, J. J., Kelly, M. H., \& DeKay, M. L. (1990). Representational momentum in memory for pitch. Journal of Experimental Psychology: Learning, Memory, and Cognition, 16(6), 1107-1117. https://doi.org/10.1037/0278-7393.16.6.1107 
Gallace, A., \& Spence, C. (2010). The science of interpersonal touch: an overview. Neuroscience and Biobehavioral Reviews, 34(2), 246-259. https://doi.org/10.1016/j.neubiorev.2008.10.004

Getzmann, S., \& Lewald, J. (2009). Constancy of target velocity as a critical factor in the emergence of auditory and visual representational momentum. Experimental Brain Research, 193(3), 437-443. https://doi.org/10.1007/s00221-008-1641-0

Getzmann, S., Lewald, J., \& Guski, R. (2004). Representational momentum in spatial hearing. Perception, 33(5), 591-599.

Goldreich, D. (2007). A Bayesian Perceptual Model Replicates the Cutaneous Rabbit and Other Tactile Spatiotemporal Illusions. PLOS ONE, 2(3), e333. https://doi.org/10.1371/journal.pone.0000333

Goldreich, D., \& Tong, J. (2013). Prediction, postdiction, and perceptual length contraction: a Bayesian low-speed prior captures the cutaneous rabbit and related illusions. Consciousness Research, 4, 221. https://doi.org/10.3389/fpsyg.2013.00221

Hall, G. S., \& Donaldson, H. H. (1885). Motor Sensations on the Skin. Mind, 10(40), 557572.

Helson, H. (1930). The Tau Effect-an Example of Psychological Relativity. Science, 71(1847), 536-537. https://doi.org/10.1126/science.71.1847.536

Hohwy, J. (2013). The predictive mind. Oxford: Oxford University Press.

Hubbard, T. L. (1990). Cognitive representation of linear motion: Possible direction and gravity effects in judged displacement. Memory \& Cognition, 18(3), 299-309.

Hubbard, T. L. (1995a). Auditory Representational Momentum: Surface Form, Direction, and Velocity Effects. The American Journal of Psychology, 108(2), 255-274. https://doi.org/10.2307/1423131 
Hubbard, T. L. (1995b). Cognitive representation of motion: evidence for friction and gravity analogues. Journal of Experimental Psychology: Learning, Memory, and Cognition, 21(1), 241.

Hubbard, T. L. (1995c). Environmental invariants in the representation of motion: Implied dynamics and representational momentum, gravity, friction, and centripetal force. Psychonomic Bulletin \& Review, 2(3), 322-338.

Hubbard, T. L. (2005). Representational momentum and related displacements in spatial memory: A review of the findings. Psychonomic Bulletin \& Review, 12(5), 822-851. https://doi.org/10.3758/BF03196775

Hubbard, T. L. (2014). Forms of momentum across space: Representational, operational, and attentional. Psychonomic Bulletin \& Review, 21(6), 1371-1403. https://doi.org/10.3758/s13423-014-0624-3

Hubbard, T. L., \& Bharucha, J. J. (1988). Judged displacement in apparent vertical and horizontal motion. Perception \& Psychophysics, 44(3), 211-221.

Hubbard, T. L., \& Motes, M. A. (2002). Does Representational Momentum Reflect a Distortion of the Length or the Endpoint of a Trajectory? Cognition, 82(3), 89-99.

Hubbard, T. L., \& Motes, M. A. (2005). An effect of context on whether memory for initial position exhibits a Fröhlich effect or an onset repulsion effect. The Quarterly Journal of Experimental Psychology. A, Human Experimental Psychology, 58(6), 961-979. https://doi.org/10.1080/02724980443000368

Intraub, H. (2004). Anticipatory spatial representation of 3D regions explored by sighted observers and a deaf-and-blind-observer. Cognition, 94(1), 19-37.

Intraub, H., Morelli, F., \& Gagnier, K. M. (2015). Visual, haptic and bimodal scene perception: evidence for a unitary representation. Cognition, 138, 132-147. https://doi.org/10.1016/j.cognition.2015.01.010 
Johnston, H. M., \& Jones, M. R. (2006). Higher order pattern structure influences auditory representational momentum. Journal of Experimental Psychology: Human Perception and Performance, 32(1), 2-17. https://doi.org/10.1037/0096-1523.32.1.2

Kennett, S., Taylor-Clarke, M., \& Haggard, P. (2001). Noninformative vision improves the spatial resolution of touch in humans. Current Biology: CB, 11(15), 1188-1191.

Kerzel, D. (2000). Eye movements and visible persistence explain the mislocalization of the final position of a moving target. Vision Research, 40(27), 3703-3715. https://doi.org/10.1016/S0042-6989(00)00226-1

Kerzel, D. (2002). A matter of design: No representational momentum without predictability. Visual Cognition, 9(1-2), 66-80.

Kerzel, D. (2003). Mental extrapolation of target position is strongest with weak motion signals and motor responses. Vision Research, 43(25), 2623-2635.

Kerzel, D., Jordan, S., \& Müsseler, J. (2001). The role of perception in the mislocalization of the final position of a moving target. Journal of Experimental Psychology: Human Perception and Performance, 27(4), 829-840. https://doi.org/10.1037/00961523.27.4.829

Langford, N., Hall, R. J., \& Monty, R. A. (1973). Cutaneous perception of a track produced by a moving point across the skin. Journal of Experimental Psychology, 97(1), 59. https://doi.org/10.1037/h0033767

Lawrence, M. A. (2016). ez: Easy Analysis and Visualization of Factorial Experiments (Version 4.4-0). Retrieved from https://cran.r-project.org/web/packages/ez/index.html

Lenggenhager, B., Loetscher, T., Kavan, N., Pallich, G., Brodtmann, A., Nicholls, M. E. R., \& Brugger, P. (2012). Paradoxical extension into the contralesional hemispace in spatial neglect. Cortex; a Journal Devoted to the Study of the Nervous System and Behavior, 48(10), 1320-1328. https://doi.org/10.1016/j.cortex.2011.10.003 
Nijhawan, R. (2002). Neural delays, visual motion and the flash-lag effect. Trends in Cognitive Sciences, 6(9), 387.

Nijhawan, R., \& Kirschfeld, K. (2003). Analogous Mechanisms Compensate for Neural Delays in the Sensory and the Motor Pathways. Current Biology, 13(9), 749-753. https://doi.org/10.1016/S0960-9822(03)00248-3

Pack, C. C., \& Bensmaia, S. J. (2015). Seeing and Feeling Motion: Canonical Computations in Vision and Touch. PLoS Biol, 13(9), e1002271. https://doi.org/10.1371/journal.pbio.1002271

Phillips, N. (2016). yarrr: A companion to the e-book YaRrr!: The Pirate's Guide to R.

R Core Team. (2013). R: A Language and Environment for Statistical Computing. Vienna, Austria. Retrieved from http://www.r-project.org/

Sarrazin, J.-C., Giraudo, M.-D., \& Pittenger, J. B. (2005). Tau and Kappa effects in physical space: the case of audition. Psychological Research, 71(2), 201-218. https://doi.org/10.1007/s00426-005-0019-1

Schlag, J., Cai, R. H., Dorfman, A., Mohempour, A., \& Schlag-Rey, M. (2000). Extrapolating movement without retinal motion. Nature, 403(6765), 38-39. https://doi.org/10.1038/47402

Seizova-Cajic, T., \& Taylor, J. L. (2014). Somatosensory Space Abridged : Rapid Change in Tactile Localization Using a Motion Stimulus. PLOS ONE, 9(3), e90892. https://doi.org/10.1371/journal.pone.0090892

Senna, I., Parise, C. V., \& Ernst, M. O. (2015). Hearing in slow-motion: Humans underestimate the speed of moving sounds. Scientific Reports, 5, 14054. https://doi.org/10.1038/srep14054

Tapley, S. M., \& Bryden, M. P. (1985). A group test for the assessment of performance between the hands. Neuropsychologia, 23(2), 215-221. 
Thornton, I. M. (2014). Representational Momentum and the Human Face: an empirical note. WICT, 2014, 101.

Tong, J., Ngo, V., \& Goldreich, D. (2016). Tactile length contraction as Bayesian inference. Journal of Neurophysiology, jn.00029.2016. https://doi.org/10.1152/jn.00029.2016

Trojan, J., Kleinböhl, D., Stolle, A. M., Andersen, O. K., Hölzl, R., \& Arendt-Nielsen, L. (2006). Psychophysical "perceptual maps” of heat and pain sensations by direct localization of CO2 laser stimuli on the skin. Brain Research, 1120(1), 106-113. https://doi.org/10.1016/j.brainres.2006.08.065

Whitsel, B. L., Favorov, O. V., Kelly, D. G., \& Tommerdahl, M. (1991). Mechanisms of dynamic peri- and intra-columnar interactions in somatosensory cortex: Stimulusspecific contrast enhancement by NMDA receptor activation. In O. Frazen \& J. Westman (Eds.), Information Processing in the Somatosensory System (pp. 353-369). London: Macmillan Press.

Whitsel, B. L., Franzen, O., Dreyer, D. A., Hollins, M., Young, M., Essick, G. K., \& Wong, C. (1986). Dependence of subjective traverse length on velocity of moving tactile stimuli. Somatosensory Research, 3(3), 185-196.

Wickham, H. (2016). tidyverse: Easily Install and Load “Tidyverse” Packages (Version 1.0.0).

Yoshikawa, S., \& Sato, W. (2008). Dynamic facial expressions of emotion induce representational momentum. Cognitive, Affective, \& Behavioral Neuroscience, 8(1), 25-31.

\section{Figures}

Figure 1. On the left: During the tactile movement stimulation participants hold their heads turned and their eyes closed. The dotted line indicates the trajectory stimulation on the lower arms, which was done by the using a long a needle. Direction, length and velocity of the 
movement were varied between trials and were presented either on the left or on the right arms. On the right: Participants indicated the felt endpoint of the trajectory with pencil while having their eyes open.

Figure 2. The interaction effect involving direction, velocity and length. The y-axis represents the distance between the perceived endpoint and the stimulated endpoint in mm (negative numbers indicate underestimation, positive numbers overestimation). For each condition the mean of each participant is presented as a dot. Moreover, the distribution for each relevant condition is depicted as well as the mean over all participants for each condition and the 95\% high density interval around the mean. 